\title{
Preparedness explains some differences between Haiti and Nepal's response to earthquake
}

In this article (BMJ 2015;350:h3059, doi:10.1136/bmj.h3059), Stanford Emergency Medicine International (http://emed. stanford.edu/education/international.html) was incorrectly referred to as "Stanford University Medicine International."
The error was introduced at the editing stage, for which we apologise.

Cite this as: BMJ 2015;350:h3160

๑ BMJ Publishing Group Ltd 2015 